\title{
A woman with visual loss, amenorrhoea and polyuria: the first reported case of nodular lymphocyte-predominant Hodgkin lymphoma presenting with hypopituitarism
}

\author{
Matthew Seymour1,3, Thomas Robertson2,3, Jason Papacostas4, Kirk Morris5, Jennifer Gillespie ${ }^{3,6}$, Debra Norris ${ }^{7}$ and \\ Emma L Duncan1,3,8
}

1Department of Endocrinology and Diabetes, ${ }^{2}$ Queensland Pathology, Royal Brisbane and Women's Hospital, Brisbane, Australia, ${ }^{3}$ Faculty of Medicine, The University of Queensland, Brisbane, Australia, ${ }^{4}$ Department of Neurosurgery, ${ }^{5}$ Department of Haematology, ${ }^{6}$ Department of Radiology, Royal Brisbane and Women's Hospital, Brisbane, Australia, ${ }^{7} \mathrm{QML}$ Pathology, Brisbane, Australia, and ${ }^{8}$ nstitute of Health and Biomedical Innovation, Faculty of Health, Queensland University of Technology, Translational Research Institute, Princess Alexandra Hospital, Brisbane Australia

Correspondence should be addressed to E L Duncan Email

emma.duncan@kcl.ac.uk

\section{Summary}

A 34-year-old woman presented 18 months post-partum with blurred vision, polyuria, amenorrhoea, headache and general malaise. Comprehensive clinical examination showed left superior temporal visual loss only. Initial investigations revealed panhypopituitarism and MRI demonstrated a sellar mass involving the infundibulum and hypothalamus. Lymphocytic hypophysitis was suspected and high dose glucocorticoids were commenced along with desmopressin and thyroxine. However, her vision rapidly deteriorated. At surgical biopsy, an irresectable grey amorphous mass involving the optic chiasm was identified. Histopathology was initially reported as granulomatous hypophysitis. Despite the ongoing treatment with glucocorticoids, her vision worsened to light detection only. Histopathological review revised the diagnosis to partially treated lymphoma. A PET scan demonstrated avid uptake in the pituitary gland in addition to splenic involvement, lymphadenopathy above and below the diaphragm, and a bone lesion. Excisional node biopsy of an impalpable infraclavicular lymph node confirmed nodular lymphocyte-predominant Hodgkin lymphoma. HyperCVAD chemotherapy was commenced, along with rituximab; fluid-balance management during chemotherapy (with its requisite large fluid volumes) was extremely complex given her diabetes insipidus. The patient is now in clinical remission. Panhypopituitarism persists; however, her vision has recovered sufficiently for reading large print and driving. To the best of our knowledge, this is the first reported case of Hodgkin lymphoma presenting initially as hypopituitarism.

\section{Learning points:}

- Lymphoma involving the pituitary is exceedingly rare and, to the best of our knowledge, this is the first reported case of nodular lymphocyte-predominant Hodgkin lymphoma presenting as hypopituitarism.

- There are myriad causes of a sellar mass and this case highlights the importance of reconsidering the diagnosis when patients fail to respond as expected to appropriate therapeutic intervention.

- This case highlights the difficulties associated with managing panhypopituitary patients receiving chemotherapy, particularly when this involves large volumes of i.v. hydration fluid. 


\section{Background}

Lymphoma involving the pituitary is exceedingly rare, with non-Hodgkin lymphoma reported in the vast majority of cases. Whilst reports of Hodgkin lymphoma involving the pituitary do exist, to the best of our knowledge, we report the first case of nodular lymphocytepredominant Hodgkin lymphoma (NLPHL) presenting as hypopituitarism.

\section{Case presentation}

A 34-year-old woman presented with 6 months of non-specific malaise, fatigue, arthralgias, headaches, blurred vision, amenorrhoea and polyuria. She had no significant past medical history. She had recently had a clinically uneventful pregnancy with delivery of her fourth child, who had been conceived without difficulty. She had breastfed her baby for 12 months during which time menses returned. Eighteen months post-partum she developed amenorrhoea, concordant with onset of malaise.

Initial evaluation elsewhere revealed anterior hypopituitarism and hyperprolactinaemia (Table 1); and whole brain MRI was reported as showing an abnormal intrasellar and suprasellar mass $(2.8 \times 1.5 \times 2.2 \mathrm{~cm})$ with thickened pituitary stalk, interpreted as consistent with

Table 1 Biochemical parameters on initial presentation.

\begin{tabular}{l}
\hline Parameters \\
\hline Sodium, mmol/L \\
Potassium, mmol/L \\
Estimated GFR, $\mathrm{LL} / \mathrm{min}$ \\
Corrected calcium, mmol/L \\
Cortisol, nmol/L \\
ACTH, ng/L \\
Free thyroxine, pmol/L \\
TSH, mIU/L \\
Estradiol, pmol/L \\
FSH, IU/L \\
LH, IU/L \\
Prolactin, mIU/L \\
Macroprolactin \\
IGF1, nmol/L \\
Serum ACE, U/L \\
LDH, U/L \\
Haemoglobin, g/L \\
WBC, $\times 109 / L$ \\
Platelets, $\times 109 / L$ \\
\hline
\end{tabular}

\begin{tabular}{c}
\hline Result \\
\hline 140 \\
4.1 \\
$>90$ \\
2.34 \\
$<35$ \\
7 \\
6.5 \\
3.6 \\
$<37$ \\
2 \\
$<0.1$ \\
3920 \\
ND \\
20 \\
194 \\
287 \\
128 \\
9.3 \\
248
\end{tabular}

\begin{tabular}{c}
\hline Normal range \\
\hline $135-145$ \\
$3.5-5.5$ \\
$>60$ \\
$2.10-2.60$ \\
$100-535$ \\
$9-51$ \\
$9-19$ \\
$0.3-3.5$ \\
$110-1650$ \\
$2-24$ \\
$2-74$ \\
$<500$ \\
$11-39$ \\
$20-70$ \\
$120-250$ \\
$115-160$ \\
$4.0-11.0$ \\
$140-400$
\end{tabular}

$\mathrm{ACE}$, angiotensin converting enzyme; $\mathrm{ACTH}$, adrenocorticotropic hormone; $\mathrm{FSH}$, follicle stimulating hormone; GFR, glomerular filtration rate; IGF1, Insulin-like growth factor 1; LDH, lactate dehydrogenase; $L H$, Luteinising hormone; ND, not detected; $\mathrm{TSH}$, thyroid stimulating hormone; WBC, white blood cell count. lymphocytic hypophysitis. She was commenced on prednisolone (50 mg daily), cabergoline, and thyroxine. However, her headaches and vision rapidly worsened and she was referred to a tertiary hospital where, on arrival, she was found to be generally unwell but afebrile and normotensive (115/75 mm Hg, without postural drop). Direct confrontation suggested only left superior temporal field loss; examination was otherwise normal with neither lymphadenopathy nor splenomegaly.

\section{Investigation}

Formal computerised perimetry was compromised by poor compliance but showed almost complete visual loss in her left eye and temporal field loss in her right eye; and dedicated pituitary MRI showed an inflammatory and infiltrative process involving the hypothalamus, optic chiasm, optic tracts, pituitary stalk and pituitary gland (Fig. 1, panel A).

\section{Treatment}

The clinical picture was thought to be most consistent with lymphocytic hypophysitis, though some clinical features (length of time post-partum) and the unusual MRI appearances beyond that usually seen in this disease process were concerning. High dose glucocorticoids and thyroxine were continued; cabergoline was ceased. Her urine output was noted to exceed 6 L daily. Serum sodium and osmolality did not exceed $143 \mathrm{mmol} / \mathrm{L}$ (135-145 mmol/L) and $294 \mathrm{mmol} / \mathrm{L}(275-295 \mathrm{mmol} / \mathrm{L})$, respectively, noting she had free access to water. She had modest steroid-induced hyperglycaemia (occasional BSL excursions, maximally to $14 \mathrm{mmol} / \mathrm{L}$ ); serum calcium was normal. Given her other clinical and biochemical features suggesting a high probability of panhypopituitarism, desmopressin 100 mcg nocte was commenced empirically, with marked diminution in urine output.

Unfortunately her vision deteriorated. After receiving i.v. methylprednisolone $500 \mathrm{mg}$ daily for 3 days followed by resumption of oral prednisone $50 \mathrm{mg}$ daily, she initially reported some visual improvement but imaging and computerised perimetry showed no change (Fig. 1, panel B) and subsequently her vision worsened again.

A subfrontal surgical biopsy revealed abnormal grey tissue directly involving the optic chiasm. Histologically, there was a mixed inflammatory cell infiltrate comprising predominantly lymphocytes and histiocytes including histiocytic aggregates, reported as granulomatous hypophysitis. The patient's vision continued to worsen 

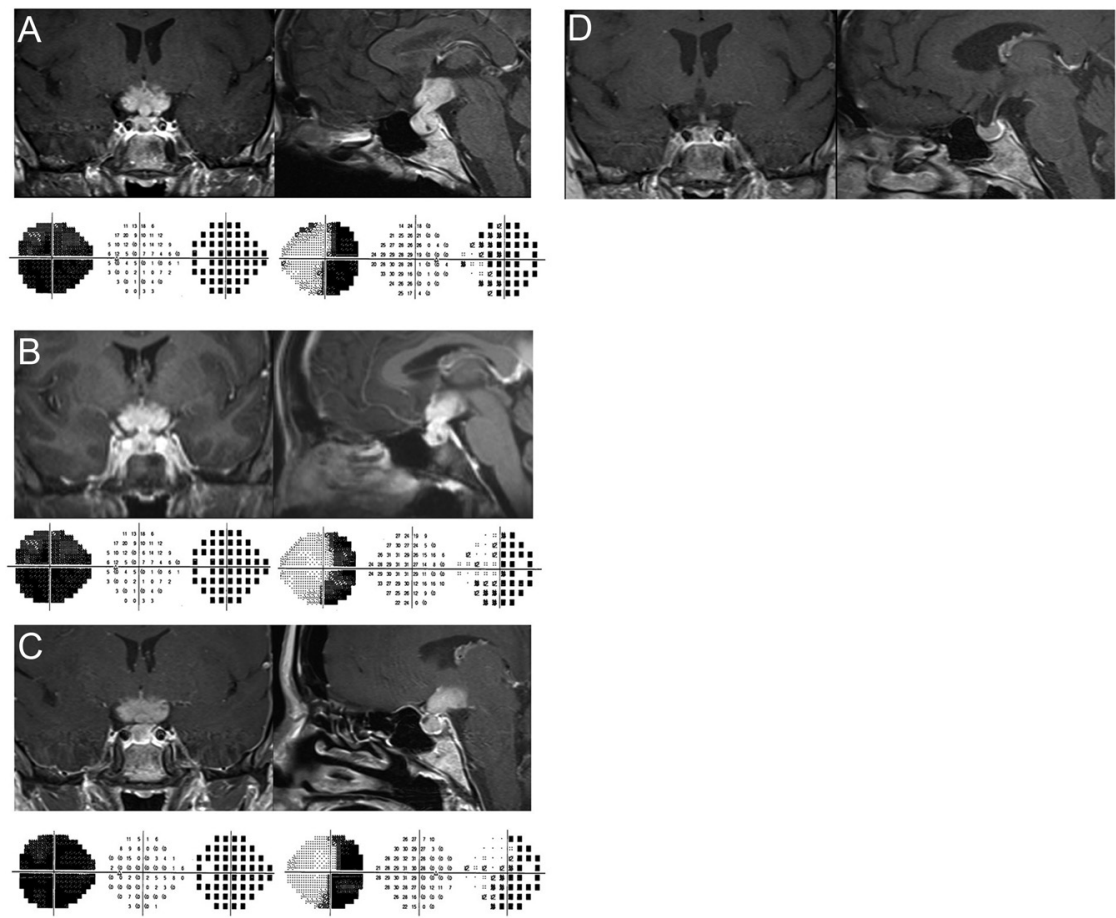

\begin{abstract}
Figure 1
Paired MRI and computerised perimetry (MRI scans are $\mathrm{T} 1$ images with gadolinium contrast) at day 5 (panel A), day 12 (panel B) and day 15 (panel C) after initial presentation. Radiological response post chemotherapy is shown in panel D.
\end{abstract}

to the point that she had difficulty distinguishing night from day. Imaging and computerised perimetry remained unchanged (Fig. 1, panel C). Neuropathological review of the biopsy identified isolated larger atypical lymphoid forms and an elevated Ki67 proliferation fraction, suggesting partially treated lymphoma.

18F-fluorodeoxyglucose positron emission tomography (FDG-PET) showed intense tracer uptake in the sellar lesion, spleen, lymph nodes both above and below the diaphragm and L3 vertebra. Excisional biopsy of an impalpable left infraclavicular lymph node demonstrated significant steroid-related changes with widespread fibrosis but in unaffected areas there were large atypical mono- and multilobate lymphoid cells in a background of small lymphocytes (Fig. 2). These cells expressed CD20, CD79a, OCT2, BOB1 and PAX5 consistent with LP cells of NLPHL. Staining for CD15 and CD30, and in situ hybridisation for EBV (EBER-ISH) was negative in the LP cells. There were varying numbers of background CD20-positive B cells, and an expanded CD21/35-positive follicular dendritic cell meshwork was present in some areas. There were more numerous background CD3positive T cells. Most of the large atypical lymphoid cells showed rosetting by $\mathrm{T}$ cells expressing PDL1, supportive of a diagnosis of NLPHL.

The patient was treated with hyper-CVAD (cyclophosphamide, vincristine, doxorubicin and dexamethasone (course A) alternating with methotrexate and cytarabine (course B)) and rituximab, requiring large volumes of i.v. hydration (course A: 9 L over 4 days; course B: 8 L over 2 days). Her concomitant diabetes insipidus made fluid management complex. Desmopressin was suspended on days of large volume loads; input was balanced against output (plus insensible losses); and she was carefully monitored both clinically and biochemically. Both hypo- and hypernatraemia were averted without needing central venous monitoring.

Post-chemotherapy FDG-PET scan showed no lymphadenopathy and normal splenic uptake. Residual L3 vertebral uptake prompted external beam radiotherapy; and residual sellar uptake was considered within physiological limits (Fig. 1, panel D).

\section{Outcome and follow-up}

Our patient is currently in complete remission. Her vision has recovered sufficiently to allow reading and driving. Panhypopituitarism persists, requiring thyroxine, cortisone acetate, a combined oral contraceptive pill, and desmopressin. Morbid obesity developed during treatment; despite resolution of Cushingoid features her BMI remained elevated. She also has poor thirst awareness, drowsiness, and hyperphagia, suggestive of hypothalamic dysfunction. Nonetheless, she underwent bariatric surgery and has subsequently lost over $30 \mathrm{~kg}$. 

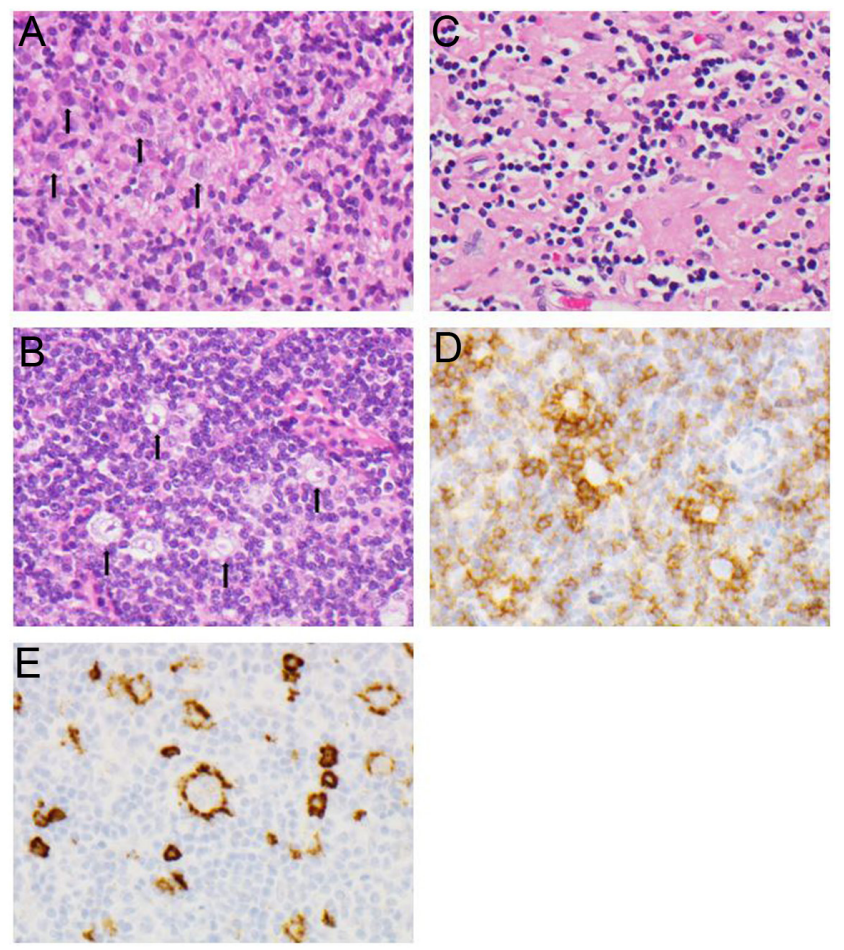

\section{Figure 2}

Pituitary and lymph node histopathology. Panel A: photomicrograph of pituitary biopsy showing isolated large atypical lymphoid cells suspicious for lymphoma cells (H\&E stain, original magnification 400x). Panel B: subsequent lymph node biopsy showing features of nodular lymphocytepredominant Hodgkin lymphoma with scattered large atypical LP cells surrounded by reactive lymphocytes (H\&E stain, original magnification 400x). Panel C: other areas of the lymph node showed significant steroid treatment effect with reduced lymphoid cells and fibrosis (H\&E stain, original magnification 400×). Panel D: PD1 immunohistochemical staining at original magnification of 400x, showing the rosetting of PD1-positive T cells around the LP cells. Panel E: positive immunoreactivity for CD20 in the LP cells $(\times 400)$.

\section{Discussion}

Involvement of the pituitary gland with any form of lymphoma is rare. The most recently published literature review identified only 35 published cases of lymphoma involving the pituitary gland (both primary and secondary involvement) with non-Hodgkin lymphoma being the vast majority (94\%) (1). Indeed, any intracranial manifestation of Hodgkin lymphoma is rare $-0.2-0.5 \%$ of cases overall $(2,3)$ - with only eight cases reported with intracranial involvement on first presentation (2). A large cohort $(n=1120)$ of patients undergoing transsphenoidal surgery for presumed pituitary adenoma identified lymphoma in only one case (4) and a separate literature review of 380 cases of metastatic disease to the pituitary gland identified only two cases of lymphoma (5).
The current WHO classification of lymphomas recognises two major types of Hodgkin lymphoma, nodular lymphocyte-predominant Hodgkin lymphoma (NLPHL) and classic Hodgkin lymphoma (CHL). We identified four cases of Hodgkin lymphoma involving the pituitary gland identified pre-mortem, none of whom presented initially with pituitary disease. Ashigbi et al. reported a case of relapsed Hodgkin lymphoma (type not specified) presenting with fever and cranial nerve palsies; biopsy of a cavernous sinus mass showed infiltration of the anterior pituitary gland by Hodgkin lymphoma. The patient did not have diabetes insipidus; and the patient was 'maintained on hydrocortisone' postoperatively with no other replacement required (6). Sapozink et al. described a case of relapsed CHL in whom headache, polyuria, polydipsia, and ocular involvement were the presenting features of relapse; CT showed a vascular lesion within the sella but at surgery and histologically Hodgkin lymphoma was reported as being 'above the sella'; no hypopituitarism was reported (3). Bunick et al. described a case of nasopharyngeal CHL eroding into the sella turcica and causing anterior pituitary insufficiency (7). In 1946, a case of Hodgkin lymphoma (type not specified) presenting with lymphadenopathy who later manifested diabetes insipidus was reported; autopsy confirmed pituitary involvement (8). The discussion listed four other cases (published in non-English journals) of 'lymphogranuloma' presenting with lymphadenopathy, three of whom developed diabetes insipidus and had pituitary involvement confirmed at autopsy and one of whom only had post-mortem evidence of pituitary involvement (8). Other cases of pituitary gland involvement by Hodgkin lymphoma unsuspected pre-mortem but identified at autopsy have been reported but only extremely rarely. One autopsy series (published 1979 in the non-English literature) of known cases of systemic lymphoma suggested pituitary involvement in approximately $25 \%$ of cases overall and up to $5 \%$ of Hodgkin lymphoma cases (9); however, pre-mortem hypopituitary symptoms and/ or biochemistry were not reported in this series (10). Some historical case reports $(3,6,7)$ provide sufficient histologic and/or immunohistochemical details to indicate that these represent variants of CHL; others lack sufficient detail to make this determination. A review of all primary lymphomas of the pituitary gland that the authors could identify (published 2001) reported no cases of HL presenting as a pituitary mass without disease evident elsewhere (10); and no case reports of NLPHL occurring in the pituitary were identified in the English literature. 
Understandably, symptoms of pituitary lymphoma have been reported more in non-Hodgkin lymphoma than Hodgkin lymphoma. These include anterior pituitary insufficiency (approx. 60\%), headache (50-60\%) and diabetes insipidus (40\%) with little apparent difference between primary and secondary disease $(1,10)$. In comparison, diabetes insipidus is the commonest clinical feature in case series of metastatic disease to the pituitary gland $(23 \%)$, followed by anterior pituitary dysfunction (12\%) and headache (8\%) (5).

The presence of diabetes insipidus complicates fluidbalance management during chemotherapy. Protocols often involve large volumes of i.v. fluid to minimise renal toxicity and reduce risk of tumour lysis syndrome. In patients receiving desmopressin, who cannot excrete a fluid load, profound hyponatraemia may ensue. Conversely, large fluid flux can reduce renal sensitivity to vasopressin through washout of the renal countercurrent mechanism. There are no guidelines for this situation. In this case, serum sodium and osmolality were maintained in the normal range with strict fluid-balance monitoring (daily weigh, twice daily electrolyte assessment, and input matched against output plus insensible losses, with adjustment as necessary (e.g. speeding up or slowing down of fluid input) as prompted by the other parameters)).

Now, 2 years since achieving complete remission, our patient remains panhypopituitary. Little is known (or reported) regarding recovery of pituitary function after treatment of lymphoma $(1,6)$. However, this looks clinically unlikely in this case.

Our patient experienced some diagnostic delay due to the initial clinical and radiographic diagnosis of lymphocytic hypophysitis and initial pathological diagnosis of granulomatous hypophysitis. Many of our patient's presenting symptoms were consistent with hypophysitis, including headache, visual disturbance, and panhypopituitarism, as were the radiological features of a symmetrical homogenously enhancing sellar mass with thickened pituitary stalk. However, the extent of the suprasellar infiltrative disease was very unusual for lymphocytic hypophysitis; and standard treatment for hypophysitis failed to elicit the expected clinical response. These prompted further diagnostic evaluation - specifically, faced with urgent clinical need, rapid deterioration, and incipient blindness, a sellar biopsy was performed. Our case highlights the importance of considering pituitary lymphoma in cases of suspected hypophysitis, particularly those with unusual features; and relevant imaging (such computerised tomography of the neck, chest, abdomen and pelvis and/or FDG-
PET scanning) should be considered in evaluating such individuals.

The initial reported pathology was granulomatous hypophysitis, which can be idiopathic, part of a clinical and pathological continuum with lymphocytic hypophysitis, or secondary to a systemic granulomatous process such as sarcoidosis, granulomatosis with polyangiitis, tuberculosis or Langerhans' cell histiocytosis. However, this too did not fully fit the clinical and radiological picture. Expert neuropathological review ultimately led to the correct clinical diagnosis and tailored management, saving her sight.

The mainstays of sellar lesion evaluation are comprehensive clinical history and examination, dedicated imaging, and hormonal testing; biopsy is rarely needed. However, it can, in rare circumstances, be the only means for a robust diagnosis - for example, in suspected malignancy, if not clinically/radiologically evident elsewhere, or in other cases where lack of response despite appropriate management for the presumed diagnosis is of grave concern, as we faced here.

In summary, this is the first case of nodular lymphocyte-predominant Hodgkin lymphoma presenting with hypopituitarism. Our case highlights the importance of reconsidering diagnoses when patients fail to respond as expected to appropriate therapeutic intervention.

\section{Declaration of interest}

The authors declare that there is no conflict of interest that could be perceived as prejudicing the impartiality of the research reported.

\section{Funding}

This research did not receive any specific grant from any funding agency in the public, commercial or not-for-profit sector.

\section{Patient consent}

Written informed consent for publication of their clinical details and clinical images was obtained from the patient.

\section{Author contribution statement}

M S, J P, K M and E D provided clinical assessment. T R and D N provided histopathological assessment and relevant images. J G provided radiological assessment and relevant images. M S and E D wrote the first draft. All authors critically reviewed the manuscript.

\section{References}

1 Koiso T, Akutsu H, Takano S, Yamamoto T, Ishikawa E, Okoshi Y \& Matsumura A. Malignant lymphoma in the parasellar region. 
Case Reports in Medicine 20142014 747280. (https://doi. org/10.1155/2014/747280)

2 Hirmiz K, Foyle A, Wilke D, Burrell S, Brownstone R, Ago C, Pahil R \& Couban S. Intracranial presentation of systemic Hodgkin's disease. Leukemia and Lymphoma 200445 1667-1671. (https://doi.org/10.108 0/10428190410001673409)

3 Sapozink MD \& Kaplan HS. Intracranial Hodgkin's disease: a report of 12 cases and review of the literature. Cancer 198352 1301-1307. (https://doi.org/10.1002/1097-0142(19831001)

4 Freda PU \& Post KD. Differential diagnosis of sellar masses. Endocrinology and Metabolism Clinics of North America 199928 81-117, vi. (https://doi.org/10.1016/s0889-8529(05)70058-x)

5 Komninos J, Vlassopoulou V, Protopapa D, Korfias S, Kontogeorgos G, Sakas DE \& Thalassinos NC. Tumors metastatic to the pituitary gland: case report and literature review. Journal of Clinical Endocrinology and Metabolism 200489 574-580. (https://doi. org/10.1210/jc.2003-030395)
6 Ashigbi MY, Venkatraj U, Agarwal V, Bello J \& Wiernik PH. Intracranial Hodgkin's disease in two patients with familial Hodgkin's disease. Medical and Pediatric Oncology 199728 255-258. (https://doi.org/10.1002/(sici)1096-911x(199704)

7 Bunick EM, Hirsh LF \& Rose LI. Panhypopituitarism resulting from Hodgkin's disease of the nasopharynx. Cancer 197841 1134-1136. (https://doi.org/10.1002/1097-0142(197803)

8 Melton EI \& McNamara WL. Hodgkin's disease involving the pituitary gland with diabetes insipidus. Annals of Internal Medicine 194625 525-531. (https://doi.org/10.7326/00034819-25-3-525)

9 Buchmann E \& Schwesinger G. Pituitary gland and haemoblastoses Zentralblatt fur Neurochirurgie 197940 35-42.

10 Giustina A, Gola M, Doga M \& Rosei EA. Clinical review 136: primary lymphoma of the pituitary: an emerging clinical entity. Journal of Clinical Endocrinology and Metabolism 200186 4567-4575. (https://doi.org/10.1210/jcem.86.10.7909)

Received in final form 25 February 2021

Accepted 23 March 2021 